\title{
PREDICTED BINDING MODE OF ANDROGRAPHOLIDE AND ITS DERIVATIVES BOUND TO PLASMODIUM FALCIPARUM GERANYLGERANYL PYROPHOSPHATE SYNTHASE
}

\author{
ANDRIANOPSYAH MAS JAYA PUTRA ${ }^{1,2}$, CHAIDIR CHAIDIR ${ }^{3}$, MUHAMMAD HANAFI ${ }^{2}$, ARRY YANUAR ${ }^{1 *}$ \\ ${ }^{1}$ Department of Pharmaceutical Chemistry, Faculty of Pharmacy, Universitas Indonesia, Depok, Indonesia. ${ }^{2}$ Department of Natural \\ Product, LIPI Research Center for Chemistry, Tangerang Selatan, Indonesia. ${ }^{3}$ Center for Pharmaceutical and Medical Technology, Agency \\ for the Assessment and Application Technology (BPPT), Tangerang Selatan, Indonesia. Email: arry.yanuar@ui.ac.id
}

Received: 21 April 2017, Revised and Accepted: 13 July 2017

ABSTRACT

Objective: Andrographolide is a major secondary metabolite in the Indonesian herb sambiloto (Andrographis paniculata). It displays a moderate antiplasmodial activity against the chloroquine-resistant strain of Plasmodium falciparum. This study aimed to investigate andrographolide inhibition of geranylgeranyl pyrophosphate synthase (GGPPS) by andrographolide molecular docking.

Methods: A comparative modeling of P. falciparum GGPPS was conducted using one of the Plasmodium vivax GGPPS crystal structures as a template. The best model from this comparative modeling was then used in a molecular docking to investigate the binding mode of andrographolide in the P. falciparum GGPPS active site.

Results: In the P. falciparum GGPPS active site, andrographolide is situated with its double rings pointing toward the hydrophobic pocket, while its lactone group is positioned between first aspartate-rich motif and second aspartate-rich motif of the catalytic pocket.

Conclusions: In the active site, andrographolide is situated with its double rings pointing toward the hydrophobic pocket, while its lactone group is positioned in the catalytic pocket.

Keywords: Andrographolide, Plasmodium falciparum, Geranylgeranyl pyrophosphate synthase, Comparative modeling, Molecular docking.

(c) 2017 The Authors. Published by Innovare Academic Sciences Pvt Ltd. This is an open accessarticle under the CC BY license (http://creativecommons. org/licenses/by/4. 0/) DOI: http://dx.doi.org/10.22159/ijap.2017.v9s1.54_60

\section{INTRODUCTION}

Plasmodium falciparum, a microorganism that belongs to the phylum Apicomplexa, is the most common cause of malaria in Indonesia [1]. The first-line treatment for uncomplicated $P$. falciparum malaria in Indonesia is the artesunate-amodiaquine combination [1]. However, a failure rate of over $10 \%$ for this treatment, associated with drug resistance, was reported in Indonesia during the period 2000-2007 [1]. The present report, therefore, offers the basis for the development of a new antimalarial drug. Geranylgeranyl pyrophosphate synthase (GGPPS) is a key enzyme in P. falciparum isoprenoid biosynthesis [2]. It catalyzes the synthesis of geranylgeranyl pyrophosphate (GGPP) [2], as well as farnesyl pyrophosphate [2], in the presence of $\mathrm{Mg}^{2+}$ cofactors [3]. This enzyme is recommended as an antimalarial drug target due to its essentiality, druggability, and amenability to high-throughput screening [4].

Andrographolide (1) is a major secondary metabolite in the Indonesian herb sambiloto (Andrographis paniculata) [5]. It has been found to have a moderate antiplasmodial activity against the chloroquineresistant strain of $P$. falciparum [6], which makes it an interesting lead for the new antimalarial drug. Since it is biosynthesized from GGPP [7], it is reasonable to hypothesize that andrographolide exerts its antiplasmodial activity by inhibition of GGPPS. To establish this hypothesis by molecular docking, however, the crystal structure of P. falciparum GGPPS is required, which is not yet available.

Meanwhile, high-resolution ligand binding crystal structures of Plasmodium vivax GGPPS are available (PDB 3CC9, 3EZ3, 3LDW, and 3PH7), based on a sequence at UniProt (at http://www.uniprot. $\operatorname{org} /$ ). A BLAST search on UniProt by for this sequence (A5K4U6) yielded a $P$. falciparum GGPPS sequence (Q86GK8) that is evidenced from transcript level. A $>70 \%$ similarity between the two sequences was revealed in our preliminary sequence alignment by ClustalX 2.1 [8]. Based on this result, we conducted a comparative modeling of $P$. falciparum GGPPS using one of the P. vivax GGPPS crystal structures as a template. The best model from this comparative modeling was then used in a molecular docking to investigate the binding mode of 1 in the $P$. falciparum GGPPS active site. This study thus aimed to investigate andrographolide inhibition on GGPPS by andrographolide molecular docking and the binding mode of the andrographolide in P. falciparum GGPPS active site.

\section{METHODS}

\section{Comparative modeling}

The comparative modeling was conducted using EasyModeller 4.0 [9]. A sequence of $P$. falciparum GGPPS, which had been downloaded from UniProt as a FASTA file (Q86GK8), was employed as the query. A highresolution ligand binding crystal structure of $P$. vivax GGPPS, which had been downloaded from the RCSB Protein Data Bank (at http:// www.pdb.org/) as a PDB file (PDB 3PH7), was selected as the template since it contains GGPP and is supported by literature [3]. During the comparative modeling, heteroatoms were not included, and loops were not refined. Instead, the model with the lowest DOPE score was optimized by default settings.

\section{Molecular docking}

The molecular docking was conducted using an AutoDock Vina module in PyRx 0.8 (At http://pyrx.sourceforge.net/) by default settings. AutoDock Vina automatically prepared protein and ligands. No water molecule was included during the molecular docking.

To investigate the binding mode of 1 in P. falciparum GGPPS, derivatives of 1 ( $2 \mathrm{a}$ and $2 \mathrm{~b}$ ) were employed. All ligand structures were drawn using $\mathrm{ACD} /$ ChemSketch 12.01 and then converted into three-dimensional structures, optimized, and saved as mol files using ACD/3D Viewer 12.01 (At http://www.acdlabs.com). The mol files were then converted into mol2 files using OpenBabel 2.3.2 [10]. ACD/ChemSketch was also used to calculate $\log \mathrm{P}$ of the ligands. 


\section{RESULTS AND DISCUSSION}

Before the comparative modeling, EasyModeller aligned sequences of the query and the template. For chain A-D of the template, the alignment scores were 265,257.6250, 273,733.8750, 252,205.1562 and 260,121.5312, respectively. Since chain B of the template gained the highest score for the alignment, and no gap presented at predicted secondary structures of the query (Supplementary Fig. 1), the comparative modeling was conducted on that chain.

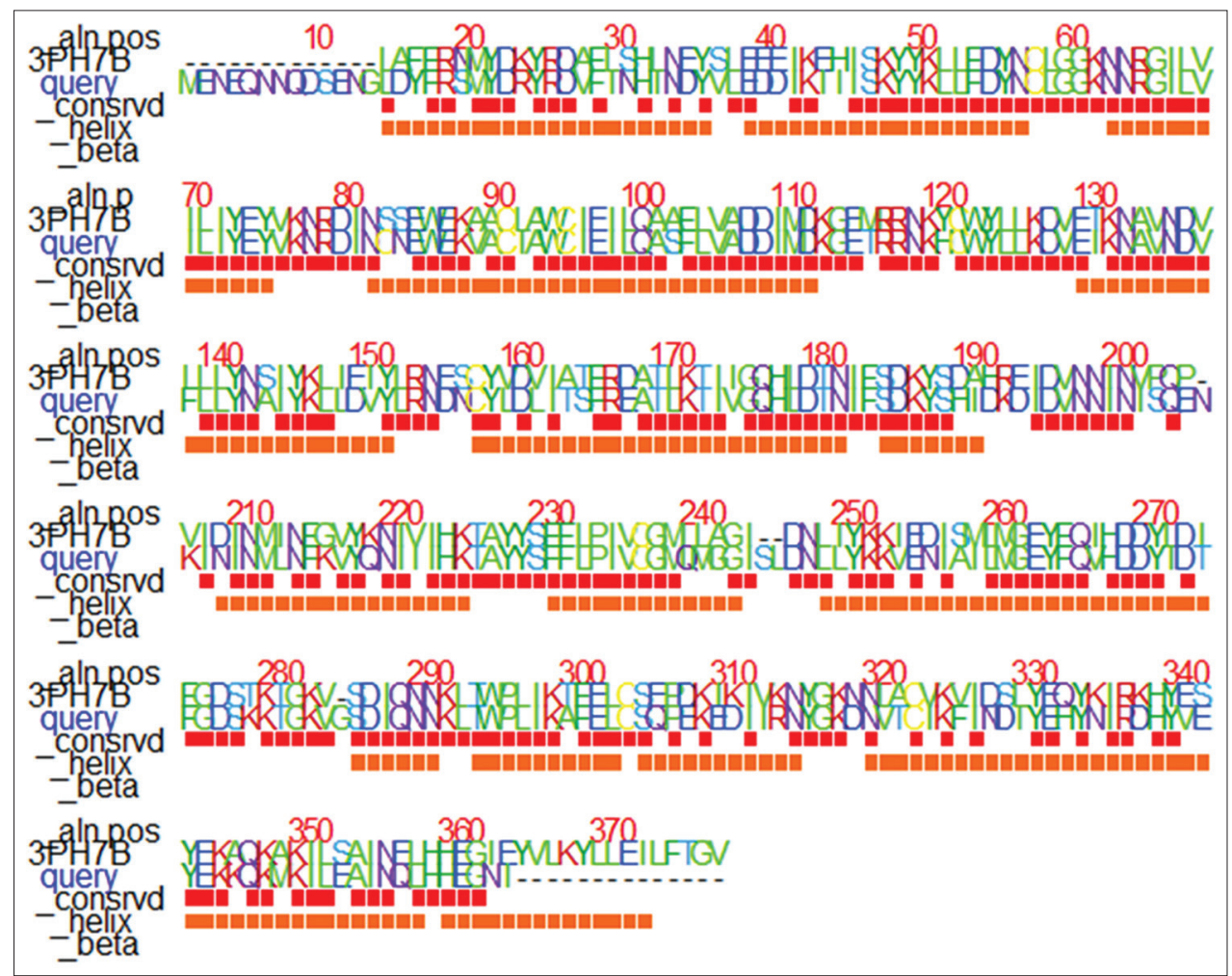

Supplementary Fig. 1: Sequence alignment between the query (Q86GK8) and template (PDB 3PH7) performed by EasyModeller 4.0 showed $70 \%$ similarity



Supplementary Fig. 2: Left: Ramachandran plot of the optimized Model 5. Right: Comparison between optimized Model 5 (red) and template (green); image captured using PyMOL Molecular Graphics System (at http://www.pymol.org/) [12] 


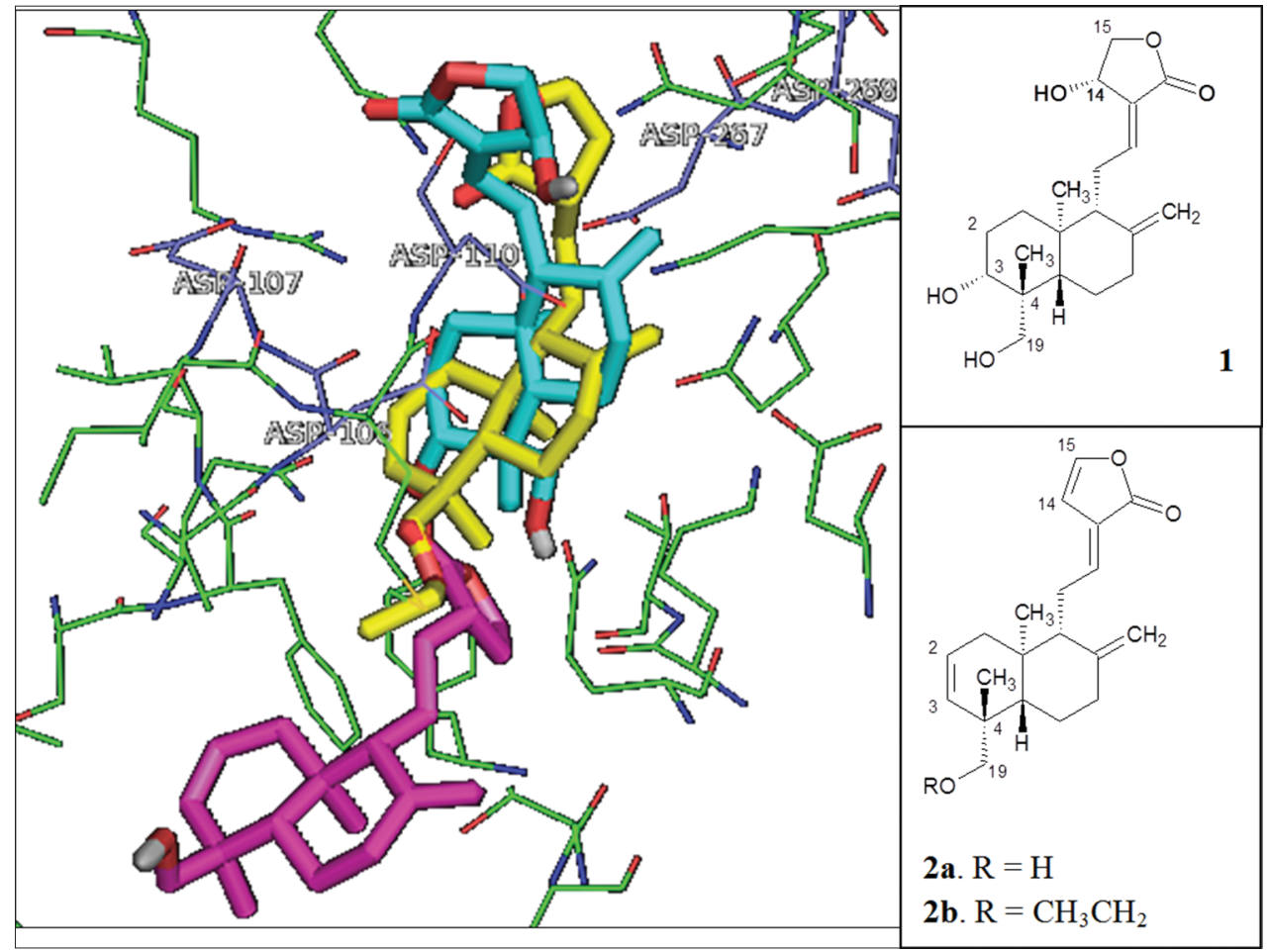

Supplementary Fig. 3: Left: Docking pose of 1 (light blue sticks), 2a (purple sticks), and 2b (yellow sticks), in Plasmodium falciparum geranylgeranyl pyrophosphate synthase model; aspartates in first aspartate-rich motif and second aspartate-rich motif depicted in blue wires and labeled; image captured using PyMOL Molecular Graphics System (at http://www.pymol.org/) [12]. Right: Structures of andrographolide (1), 2a, and $2 b$

The comparative modeling produced seven models with the following DOPE scores $-45,876.36719,-45,724.93750,-45,170.59375$, $-45,537.06250,-45,908.73047,-45,727.52344$, and $-45,883.46484$. Model 5 had the lowest DOPE score, so it was optimized. After optimization of this model, a Ramachandran plot revealed only a few residues outside the allowed regions $\left(G^{79}, G^{300}\right.$, and $S^{304}$; Supplementary Fig. 2). The residues were not parts of the presumed P. falciparum GGPPS active site. A comparison between this model and the template is presented in Fig. 2.

The active site of $P$. vivax GGPPS consists of two pockets: a hydrophobic anchor $\left(\mathrm{L}^{118}, \mathrm{Q}^{119}, \mathrm{~A}^{120}, \mathrm{~F}^{122}, \mathrm{~V}^{156}, \mathrm{Y}^{160}, \mathrm{~T} 188\right.$, and $\left.\mathrm{I}^{192}\right)$ and a catalytic pocket, which contains the first aspartate-rich motif (FARM; ${ }^{126} \mathrm{DDIMD}^{130}$ ) and second aspartate-rich motif (SARM; ${ }^{287}{ }^{D D Y I D}{ }^{291}$ ) [3]. GGPP positions itself in the $P$. vivax GGPPS active site such that its hydrophobic part is held by the anchor, while its pyrophosphate group interacts with the catalytic pocket, either directly or through $\mathrm{Mg}^{2+}$ cofactors and/or water molecules [3]. A similar position and orientation were confirmed for 1 in the P. falciparum GGPPS active site by our molecular docking. The double rings of 1 were pointing toward $\mathrm{F}^{122}, \mathrm{~T}^{188}$, and $\mathrm{I}^{192}$ of the hydrophobic pocket, while its lactone group was positioned between FARM and SARM of the catalytic pocket (Supplementary Fig. 3). Although the lactone group did not interact strongly with the FARM and SARM, stronger interactions should be expected in the presence of $\mathrm{Mg}^{2+}$ cofactors and water molecules. This finding suggests the double rings of 1 are essential for hydrophobic interactions, while its lactone group is essential for hydrophilic ones.

The $\log P$ of 1 is low (1.62) and its bioavailability is also low [11]. Thus, we proposed a more hydrophobic analog of 1 by the elimination of its hydroxyl groups at $\mathrm{C}-3$ and $\mathrm{C}-14(2 \mathrm{a} ; \log \mathrm{P}=3.70)$. This analog, when docked into our P. falciparum GGPPS model, left the catalytic pocket and positioned itself close to $\mathrm{F}^{122}, \mathrm{~T}^{188}$, and $\mathrm{I}^{192}$ of the hydrophobic pocket (Supplementary Fig. 3). However, when this analog was alkylated at the $\mathrm{C}-19$ hydroxyl group $(2 \mathrm{~b} ; \log \mathrm{P}=4.91)$, the original position and orientation were restored (Supplementary Fig. 3).
Molecule 1 did not fill most of the P. falciparum GGPPS hydrophobic pocket. This explains its moderate inhibitory activity. An alkylation strategy for 1 , as demonstrated by $2 \mathrm{~b}$, would enhance its coverage on the hydrophobic pocket at the expense of its $\log P$.

\section{CONCLUSION}

In the P. falciparum GGPPS active site, andrographolide is situated with its double rings pointing toward the hydrophobic pocket, while its lactone group is positioned between FARM and SARM of the catalytic pocket.

\section{REFERENCES}

1. Elyazar IR, Hay SI, Baird JK. Malaria distribution, prevalence, drug resistance and control in Indonesia. Adv Parasitol 2011;74: 141-75.

2. Jordão $\mathrm{FM}$, Gabriel $\mathrm{HB}$, Alves JM, Angeli CB, Bifano TD, Breda A, et al. Cloning and characterization of bifunctional enzyme farnesyl diphosphate/geranylgeranyl diphosphate synthase from Plasmodium falciparum. Malar J 2013;12:184

3. Artz JD, Wernimont AK, Dunford JE, Schapira M, Dong A, Zhao Y, et al. Molecular characterization of a novel geranylgeranyl pyrophosphate synthase from Plasmodium parasites. J Biol Chem 2011;286(5):3315-22.

4. Crowther GJ, Napuli AJ, Gilligan JH, Gagaring K, Borboa R, Francek $\mathrm{C}$, et al. Identification of inhibitors for putative malaria drug targets among novel antimalarial compounds. Mol Biochem Parasitol 2011:175(1):21-9.

5. Pholphana N, Rangkadilok N, Saehun J, Ritruechai S, Satayavivad J. Changes in the contents of four active diterpenoids at different growth stages in Andrographis paniculata (Burm.f.) Nees (Chuanxinlian). Chin Med 2013;8(2):1-12

6. Mishra K, Dash AP, Dey N. Andrographolide: A novel antimalarial diterpene lactone compound from Andrographis paniculata and its interaction with curcumin and artesunate. J Trop Med 2011;2011:579518.

7. Srivastava N, Akhila A. Biosynthesis of andrographolide in Andrographis paniculata. Phytochemistry 2010;71(11-12):1298-304. 
8. Larkin MA, Blackshields G, Brown NP, Chenna R, McGettigan PA, McWilliam $\mathrm{H}$, et al. Clustal W and Clustal X version 2.0. Bioinformatics 2007;23:2947-8.

9. Kuntal BK, Aparoy P, Reddanna P. EasyModeller: A graphical interface to MODELLER. BMC Res Notes 2010;3:226.

10. O’Boyle NM, Banck M, James CA, Morley C, Vandermeersch T, Hutchison GR. Open Babel: An open chemical toolbox. J Cheminform
2011;3:33

11. Ye L, Wang T, Tang L, Liu W, Yang Z, Zhou J, et al. Poor oral bioavailability of a promising anticancer agent andrographolide is due to extensive metabolism and efflux by P-glycoprotein. J Pharm Sci 2011;100(11):5007-17.

12. DeLano WL. The PyMOL Molecular Graphics System. Palo Alto, CA: DeLano Scientific; 2008 